\title{
DCT Coding of Nonrectangularly Sampled Images
}

\author{
Emre Gündüzhan, A. Enis Çetin, and A. Murat Tekalp
}

\begin{abstract}
Discrete cosine transform (DCT) coding is widely used for compression of rectangularly sampled images. In this letter, we address efficient DCT coding of nonrectangularly sampled images. To this effect, we discuss an efficient method for the computation of the DCT on nonrectangular sampling grids using the Smith-normal decompesition. Simulation results are provided.
\end{abstract}

\section{INTRODUCTION}

I N DIGITAL representation of multidimensional (M-D) signals, various sampling structures can be used that are usually in the form of a lattice or a union of coset of a lattice [1]. Rectangular sampling grids (orthogonal lattices) are the most commonly used lattice structure. It is well known that the sampling efficiency of a lattice depends on the region of support of the spectrum of the analog signal [2]; [3]. In still images, a 2-D nonrectangular sampling grid may require a smaller number of samples per unit area than a rectangular grid. In video, interlaced sampling grids (3-D nonrectangular grids) are widely used for reduced flickering without increasing the transmission bandwidth.

Most algorithms currently used for processing and compression of nonrectangularly sampled signals do not fully exploit the nature of the sampling structure used. The standard practice to compress nonrectangularly sampled still images by JPEGtype schemes is to first interpolate the image to a rectangular grid, which usually has twice as many pixels. Alternatively, nonrectangularly sampled images can be treated as if they were sampled on a rectangular grid, but this would cause artificial high frequencies to appear in the DCT representation since horizontal and vertical lines in the image will be distorted. Thus, the latter choice usually results in a poorer compression efficiency. Likewise in video compression, the frame DCT option in MPEG-II assumes that the even and the odd fields were recorded at the same time instant on a rectangular grid, which may introduce artificial high-frequency components.

In this paper, we address DCT coding of images sampled on arbitrary lattices. To this effect, we discuss an efficient method for the computation of the DCT on nonrectangular sampling grids, which is similar to the computation of the MD DFT in nonrectangular grids [2], [4]. The method is based on the Smith-normal decomposition, that is, diagonalizing the periodicity matrix of the M-D sequence. The original sequence

Manuscript received December 14, 1993; approved May 19, 1994. This work was supported by TUBITAK and NATO (900012). The associate editor coordinating the review of this letter and approving it for publication was Prof. H. J. Trussell.

E. Gündüzhan and A. E. Cetin are with the Electrical and Electronics Engineering Department, Bilkent University, Bilkent, Ankara, Turkey.

A. M. Tekalp is with the Electrical Engineering Department, University of Rochester, Rochester, NY, 14627, USA.

IEEE Log Number 9405349.

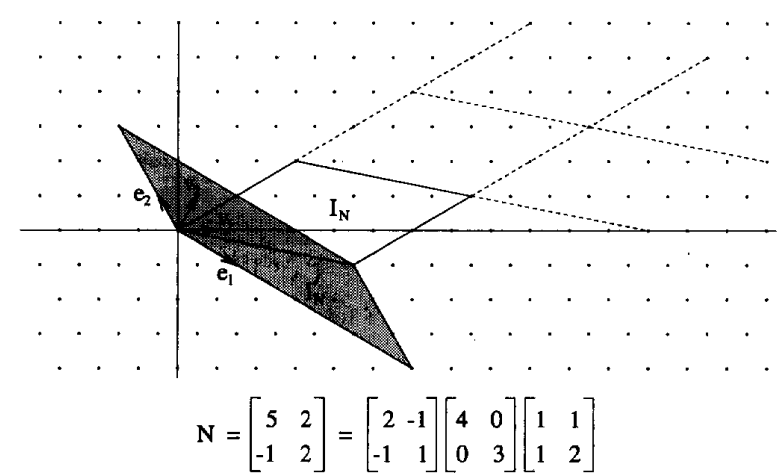

Fig. 1. Coordinate transformation in a hexagonal lattice.

is first transformed onto a new coordinate system, where it is rectangularly periodic. Rectangular M-D DCT of the transformed sequence is computed in the new coordinates, and the result is transformed back to the original coordinates. A short review of the notation and the Smith-normal decomposition is given in Section II. In Section III, an efficient method to compute the DCT and the inverse DCT in nonrectangular grids is described. Finally, in Section IV, simulation results are presented for quincunx sampling grids [5]-[10].

\section{PREliminaries}

Let $\tilde{x}(\mathbf{n})$ be a periodic sequence, i.e.

$$
\tilde{x}(\mathbf{n}+\mathbf{N r})=\tilde{x}(\mathbf{n}) \quad \forall \mathbf{n}, \mathbf{r} \in Z^{D}
$$

where the matrix $\mathbf{N}$ is a nonsingular integer matrix, and it is called the periodicity matrix. The number of samples in one period for a given periodicity matrix $\mathbf{N}$ is unique and given by $|\operatorname{det} \mathbf{N}|$. The periodicity matrix $\mathbf{N}$ is not unique because $\hat{\mathbf{N}}=\mathbf{N L}$ represents the same periodicity if $\mathbf{L}$ is a unimodular ( $|\operatorname{det} L|=1$ ) integer matrix [4].

Let $\mathbf{v}_{1} \cdots \mathbf{v}_{D}$ be linearly independent real vectors in a $D$ dimensional Euclidean space. A lattice $\Lambda$ in $R^{D}$ is the set of all linear combinations of $\mathbf{v}_{1} \cdots \mathbf{v}_{D}$ with integer coefficients [4].

$\Lambda=\left\{n_{1} \mathbf{v}_{1}+n_{2} \mathbf{v}_{2}+\cdots+n_{D} \mathbf{v}_{D} \mid n_{i} \in Z, \quad i=1, \ldots, D\right\}$.

The matrix $\mathbf{V}=\left[\mathbf{v}_{1} \mathbf{v}_{2} \cdots \mathbf{v}_{D}\right]$ is called the sampling matrix of the lattice, which is not unique. If $\mathbf{E}$ is any unimodular matrix, then $\hat{\mathbf{V}}=\mathbf{V E}$ is also a sampling matrix for the same lattice. However, $|\operatorname{det} V|$ is unique for a given lattice, and it is called the sampling density [1].

Any nonsingular integer matrix $\mathbf{N}$ can be diagonalized by pre and postmultiplication by unimodular integer matrices $\mathbf{E}$ 


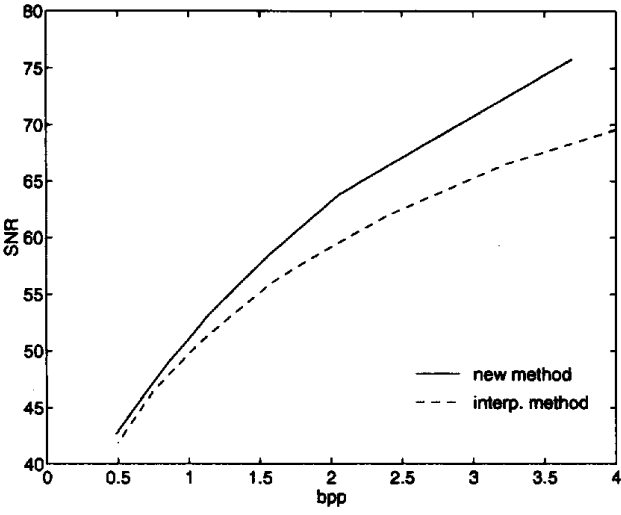

Fig. 2. Comparison of the two methods for the Barbara image.

TABLE I

COMPRESSION RESULTS FOR BARBARA IMAGE

\begin{tabular}{||l|c|c|c||}
\hline & Compression Ratio & MSDE & SNR \\
\hline \hline $3.69 \mathrm{bpp}$ & 2.17 & 1.74 & 75.79 \\
\hline $2.06 \mathrm{bpp}$ & 3.88 & 3.46 & 63.85 \\
\hline $1.57 \mathrm{bpp}$ & 5.09 & 4.70 & 58.53 \\
\hline $1.32 \mathrm{bpp}$ & 6.07 & 5.61 & 55.45 \\
\hline $1.13 \mathrm{bpp}$ & 7.05 & 6.43 & 53.06 \\
\hline $1.01 \mathrm{bpp}$ & 7.96 & 7.18 & 51.17 \\
\hline $0.86 \mathrm{bpp}$ & 9.30 & 8.12 & 49.03 \\
\hline $0.70 \mathrm{bpp}$ & 11.46 & 9.44 & 46.39 \\
\hline $0.49 \mathrm{bpp}$ & 16.22 & 11.69 & 42.68 \\
\hline
\end{tabular}

TABLE II

COMPRESSION RESULTS FOR LENA IMAGE

\begin{tabular}{||l|c|c|c||}
\hline & Compression Ratio & MSDE & SNR \\
\hline \hline $3.09 \mathrm{bpp}$ & 2.59 & 1.70 & 75.49 \\
\hline $1.58 \mathrm{bpp}$ & 5.06 & 2.94 & 66.04 \\
\hline $1.17 \mathrm{bpp}$ & 6.85 & 3.69 & 62.08 \\
\hline $0.96 \mathrm{bpp}$ & 8.35 & 4.19 & 59.87 \\
\hline $0.82 \mathrm{bpp}$ & 9.75 & 4.60 & 58.23 \\
\hline $0.73 \mathrm{bpp}$ & 11.00 & 4.98 & 56.88 \\
\hline $0.62 \mathrm{bpp}$ & 12.82 & 5.44 & 55.32 \\
\hline $0.51 \mathrm{bpp}$ & 15.61 & 6.15 & 53.20 \\
\hline $0.38 \mathrm{bpp}$ & 21.12 & 7.48 & 49.80 \\
\hline
\end{tabular}

and $\mathbf{F}$, respectively, such that [4], [10]

$$
\mathbf{N}=\mathbf{E} \boldsymbol{\Lambda} \mathbf{F}
$$

where i) $\Lambda$ is in Smith-normal form (or elementary divisor form), i.e, $\boldsymbol{\Lambda}$ is diagonal and $\Lambda_{11}\left|\Lambda_{22}\right| \cdots\left|\Lambda_{D D}\right|$ ("|" denotes "divides"), and ii) $|\operatorname{det} E|=|\operatorname{det} \mathbf{F}|=1$. Such a decomposition, which is called the Smith-normal decomposition, can be

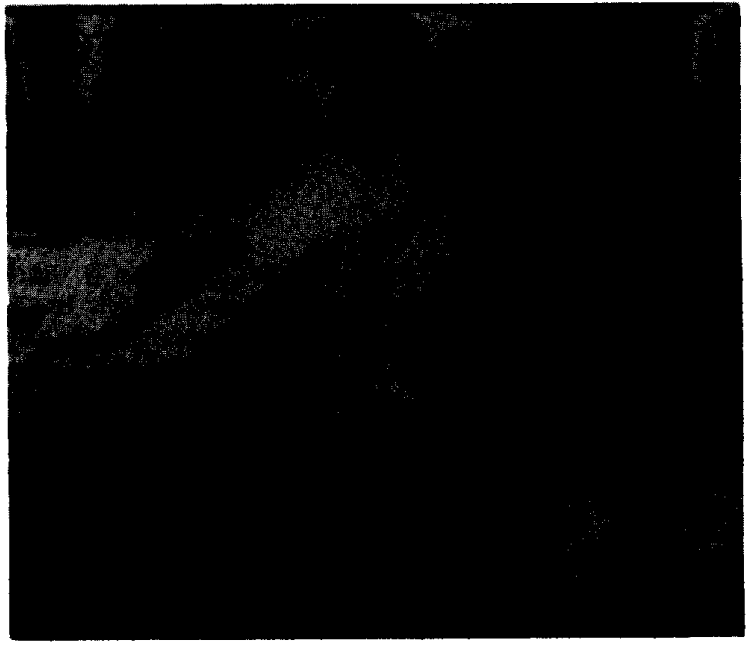

(a)

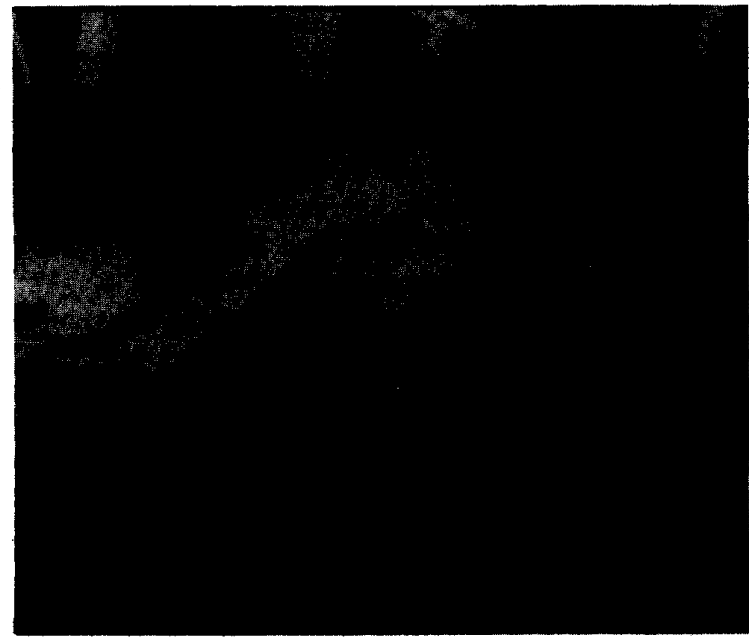

(b)

Fig. 3. Original (a) and reconstructed (b) Barbara images of size $(336 \times 560)$ in a quincunx sampling grid $(1.57 \mathrm{bpp}, \mathrm{SNR}=58.53)$.

used for transforming an arbitrary periodic sampling grid to a rectangular grid.

\section{COMPUTATION OF DCT IN NONRECTANGULAR GRIDS}

The sequence will be first transformed to a new coordinate system where the periodicity matrix is diagonal. The DCT will be computed in the rectangular coordinate system, and the result will be transformed back to the original coordinates.

Let $x(\mathbf{n})$ be a finite-extent M-D sequence with a support $I_{N}$. Let $\tilde{x}(\mathbf{n})$ denote its periodic extention with a periodicity matrix $\mathbf{N}$, which can always be decomposed as in (3). The sequence $\tilde{x}(\mathbf{n})$ can be reordered using the coordinate transformation

$$
\mathbf{n}^{\prime}=\mathbf{E}^{-1} \mathbf{n} \text {. }
$$

Let us denote the sequence in the new coordinates by $\tilde{x}^{\prime}\left(\mathbf{n}^{\prime}\right)$. It can be shown that $\tilde{x}^{\prime}\left(\mathbf{n}^{\prime}\right)$ is periodic with $\Lambda$ in the primed coordinates. 
An example of the coordinate transformation is shown in Fig. 1 in a hexagonal sampling structure (for $D=2$ ), where $\mathbf{v}_{1}$ and $\mathbf{v}_{2}$ are two basis vectors, and $I_{N}$ is the fundamental period for the periodicity matrix $\mathbf{N}$. The basis vectors become $\mathbf{e}_{1}$ and $\mathbf{e}_{2}$ after the coordinate transformation, and $I_{N}^{\prime}$ is the fundamental period for the new periodicity matrix that is diagonal.

The algorithm to compute the DCT of a sequence $x(\mathbf{n})$ can be summarized as follows:

i. Compute the transformation $\mathbf{n}^{\prime}=\mathbf{E}^{-1} \mathbf{n}$, and find the sequence in the new coordinates $x^{\prime}\left(\mathbf{n}^{\prime}\right)$.

ii. Compute the rectangular DCT of $x^{\prime}$ to obtain $X^{\prime}\left(\mathbf{k}^{\prime}\right)$.

iii. Compute the back transformation $\mathbf{k}=\mathbf{F}^{T} \mathbf{k}^{\prime}$ to get $X(\mathbf{k})$.

In order to compute the inverse DCT, the steps are reversed:

i. Compute the transformation $\mathbf{k}^{\prime}=\left(\mathbf{F}^{-1}\right)^{T} \mathbf{k}$, and find $X^{\prime}\left(\mathbf{k}^{\prime}\right)$.

ii. Compute the rectangular inverse DCT to get $x^{\prime}\left(\mathbf{n}^{\prime}\right)$.

iii. Find $x(\mathbf{n})$ using $\mathbf{n}=\mathbf{E n}^{\prime}$.

If $I_{N}$ is the support of $x(\mathbf{n})$, then $I_{N}$ should be mapped into the region $I_{N}^{\prime}=\left[0, \Lambda_{11}\right) \times\left[0, \Lambda_{22}\right)$ for the DCT (or DFT) computation to be possible. Since DFT uses a periodicity matrix of $\Lambda, I_{N}$ is always mapped into $I_{N}^{\prime}$. In the case of the DCT computation, the periodicity matrix is $2 \Lambda$, and due to this fact, it is not possible to map $I_{N}$ into $I_{N}^{\prime}$ for all choices of the sampling and periodicity matrices. However, it can be shown that the DCT can be defined for all sampling lattices by properly choosing the (nonunique) sampling and the periodicity matrices for the given lattice.

\section{Simulation Results}

In our experiments, we have compared the proposed method with the method of interpolating to a denser grid and then using the standard JPEG algorithm. Images sampled on a quincunx lattice are first divided into $8 \times 8$ blocks. Then, the DCT of each block is computed using our new algorithm. The quantized transform domain coefficients are converted into a bit stream by using the JPEG entropy encoder. The compression results for the Barbara and Lena images are shown in Tables I and II, respectively. The original Barbara image and the compressed/decompressed Barbara image with a compression ratio of 5.09 are shown in Fig. 3.
The same images are also coded by interpolating the quincunx grid to a denser rectangular grid (with twice as many samples). The standard JPEG algorithm is then applied. At the receiver, the incoming bit stream is decoded to get a rectangularly sampled image that is decimated back to the quincunx grid. The comparison of the compression results using the Barbara image is shown in Fig. 2. It can be seen that the new procedure proposed in this letter provides better results at all coding levels. For example, in the case of the Barbara image compressed to $1.57 \mathrm{bpp}$, the SNR of the compressed/decompressed image with the new method is 58.53, whereas interpolation to rectangular grid first yields the SNR 55.83 .

\section{CONCLUSION}

In this letter, the discrete cosine transform (DCT) is defined on nonrectangular sampling grids, and a DCT-based compression scheme for nonrectangularly sampled images is presented. We note that the DCT can be defined for all lattices by the proper choice of the sampling and periodicity matrices.

\section{REFERENCES}

[1] E. Dubois, "The sampling and reconstruction of time-varying imagery with application in video systems," Proc. IEEE, vol. 73, pp. 502-522, Apr. 1985.

[2] D. E. Dudgeon and R. M. Mersereau, Multidimensional Digital Signal Processing. Englewood Cliffs, NJ: Prentice-Hall, 1984.

[3] G. J. Tonge, "The sampling of television images," Int. Broadcasting Assoc. (IBA) Rep., 112/81.

[4] M. A. Rahgozar and J. P. Allebach, "A general theory of time-sequential sampling," Signal Processing, Sept. 1992.

[5] A. E. Çetin, "A multiresolution nonrectangular wavelet representation for two-dimensional signals," Signal Processing, vol. 32, no. 3, June 1993.

[6] R. Ansari and C. L. Lau, "Two-dimensional IIR filters for exact reconstruction in tree-structured sub-band decomposition," Electron. Lett., vol. 23, pp. 633-634, June 1987.

[7] R. Ansari, A. E. Cetin, and S. Lee, "Subband coding of images using nonrectangular filter banks," Proc. SPIE Conf. Applications Image Processing XI (San Diego), Aug. 1988.

[8] J. Kovacevic and M. Vetterli, "Nonseparable multidimensional perfect reconstruction filter banks and wavelet for $R^{n}$," IEEE Trans. Inform. Theory, vol. 38, pp. 533-555, Mar. 1992.

[9] M. Barlaud et. al., "Pyramidal lattice vector quantization for multiscale image coding," submitted to IEEE Trans. on Image Processing.

[10] M. Pohst and H. Zassenhaus, Algorithmic Algebraic Number Theory. Cambridge, UK: Cambridge University Press, 1989. 